\title{
Recurrent pericardial effusion: the value of polymerase chain reaction in the diagnosis of tuberculosis
}

\author{
B S Rana, R A Jones, I A Simpson
}

\begin{abstract}
A 23 year old army man presented with progressive dyspnoea and was found to have a massive pericardial effusion. Despite extensive investigations the cause remained elusive, until samples were sent for polymerase chain reaction (PCR). This case was unusual for several reasons and is a reminder of the atypical way in which tuberculosis infection can present and how a high index of suspicion should be maintained. It shows the importance of molecular biological advances in providing simple and rapid methods for arriving at the correct diagnosis, by way of nucleic acid probes and polymerase chain reaction.

(Heart 1999;82:246-247)
\end{abstract}

Keywords: polymerase chain reaction; tuberculous pericarditis; pericardial effusion

A 23 year old army tuba player presented with mild but progressive dyspnoea and a vague history of dysphagia and epigastric discomfort. Six months previously, he had contracted a lower respiratory tract infection. His medical history included BCG vaccination. Two years earlier he had served in Bosnia. Although air entry was reduced at the left base, examination was unremarkable. The chest $x$ ray showed a globular cardiac shadow with clear lung fields. Echocardiography showed a massive pericardial effusion with evidence of right atrial diastolic collapse (fig 1). At pericardiocentesis, three litres of bloodstained fluid were drained.

The fluid contained an abundance of lymphocytes but microbiology and cytology were negative. Full blood count, renal function, bone profile, clotting studies, and liver function tests were normal. Inflammatory markers (erythrocyte sedimentation rate $(\mathrm{ESR}) / \mathrm{C}$ reactive protein), viral serology (including influenza A and B, streptococcus, Borrelia burgdorferi, mycoplasma, chlamydia, respiratory syncitial virus, adenovirus, and hepatitis B antibodies), microbiology (including syphilis), and autoimmune and malignancy screens (CA 125, human chorionic gonadotrophin, carcinoembryonic antigen, $\alpha$ fetoprotein, lactase dehydrogenase $(\mathrm{LDH})$ ) were all unremarkable. Thy- roid function was normal. A mantoux test was positive at 1:1000, consistent with previous BCG vaccination, while alcohol acid fast bacilli (AAFB) stain and special culture for tuberculosis were found to be negative. The patient was HIV negative.

Computed tomograpy (CT) of the chest revealed a bulky anterior and superior mediastinum but no lymphadenopathy. Three weeks after the initial procedure the pericardial effusion reaccumulated and needed further drainage. Bronchoscopy and mediastinoscopy with pericardial biopsy were performed, and a pericardial window made. Despite this intervention the effusion reaccumulated for the third time. The pericardial biopsy showed non-specific changes.

Further high resolution spiral CT scanning of the chest revealed areas of pericardial calcification posterior to the left ventricle, and small calcified nodes in the lower left hilum and paraoesophageal region. The mediastinum appeared to be oedematous. Lymphoma was considered unlikely because of the full blood count and normal LDH and ESR, and lack of clinical signs and symptoms. The radiological appearances suggested chronic mediastinitis, and, in view of the tissue calcification, tuberculosis infection was considered likely. Because of the results of the CT scan, pericardial fluid was sent for polymerase chain reaction (PCR); the test for Mycobacterium tuberculosis infection was positive. Thus, tuberculous pericarditis was diagnosed and the patient started antituberculous

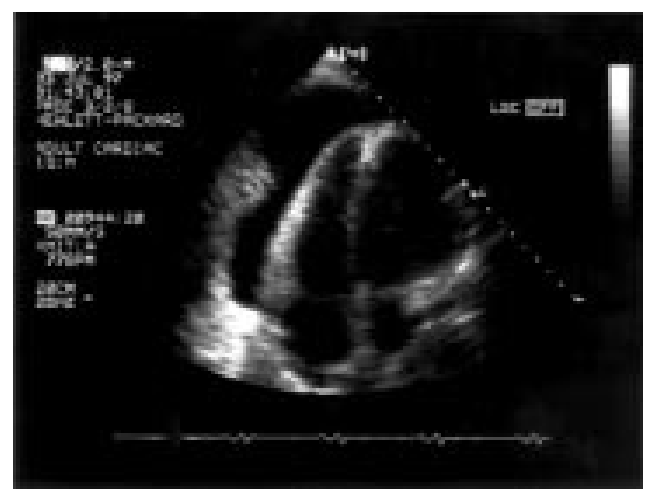

Figure 1 Transthoracic echocardiogram showing a massive pericardial effusion, seen here in the apical four chamber view. 
treatment (rifampicin, isoniazid, pyrazinamide, and pyridoxine) with prednisolone as adjunctive steroid treatment. The patient remained well during this period but pericardial fluid continued to accumulate and a second pericardial window was made, via a limited sternotomy. The second PCR result also tested positive for tuberculosis. Two weeks after starting treatment, transthoracic echocardiography showed only a minimal amount of fluid in the pericardial space. One month later the steroid dose was reduced, and during this time the patient returned to work. He will continue antituberculosis treatment for nine months.

\section{Discussion}

In industrialised countries, the incidence of tuberculous pericarditis has declined over the past 40 years. ${ }^{1}$ In the United Kingdom it is now more common, particularly as a consequence of immunosuppression, and is seen frequently in patients with AIDS. Spread to the pericardium occurs most often by local extension from hilar and mediastinal lymph nodes. ${ }^{2}$ Tuberculous pericarditis may also be seen in association with pulmonary or disseminated tuberculosis. In patients with pulmonary disease, the incidence of concomitant pericardial disease ranges from $1-8 \% .{ }^{1}$ Often, patients will present with non-specific signs - such as, fever, night sweats, fatigue, and dyspnoea. Pericardial effusion usually follows.

Our patient was HIV seronegative, and systemic upset was absent; this was an unusual presentation in an otherwise fit young man. $\mathrm{He}$ played the tuba in the army band, and he was probably exposed to the disease when he was stationed in Bosnia two years earlier.

It is notoriously difficult to isolate $M$ tuberculosis from pericardial fluid. AAFB smears are usually negative, but culture is positive in $50-86 \%$ of cases. $^{2}$ Our case shows the importance of PCR in arriving at the correct diagnosis. Advances in molecular techniques have created methods that aid identification of the presence of infectious agents that have been difficult to isolate previously. The use of DNA probes with amplification and hybridisation of

\section{Key points}

This case was remarkable because:

- It was an unusual presentation because of the lack of constitutional upset expected with active tuberculosis infection, importantly, the absence of fever and night sweats

- The patient was HIV seronegative, which is an atypical finding in a patient with these symptoms and with no underlying immunosuppression

- Despite repeated testing, the culture was negative for tuberculosis

- It shows the importance of molecular biological advances in providing relatively simple and rapid methods for reaching a correct diagnosis

tuberculosis DNA which is present in clinical samples, such as pericardial fluid, has been validated and allows the disease to be diagnosed earlier. ${ }^{3}$ More recently, the use of reverse transcriptase PCR as a more accurate guide to the identification of viable $M$ tuberculosis has been explored, and in the future may improve diagnostic sensitivity. ${ }^{4}$

Interestingly, pericardial biopsy was unhelpful in this case but reports suggest the yield to be in the range of $83-100 \%$ in HIV negative patients ${ }^{2}$; this does not exclude a diagnosis of tuberculosis, as this case shows. Recently, raised adenosine deaminase activity has been useful in diagnosing tuberculosis (in circumstances where connective tissue and lymphoproliferative disorders can be excluded). ${ }^{5}$ Survival rates have been shown to improve with the use of adjunctive steroid treatment, ${ }^{6}$ reducing mortality and morbidity from complications such as constrictive pericardial disease.

We acknowledge Dr G Sterling's contribution to this case.

1 Lorell BH. Pericardial diseases. In: Braunwald E, ed. Heart disease $5^{\text {th }}$ ed. Philadelphia: W B Saunders 1997:1507-8.

2 Muthuswamy P, Tan T, Cohen R. Sudden onset of large pericardial effusion in a 27 year old man with AIDS. Chest 1995;108: 1739-41.

3 Akcan Y, Tuncer S, Hayran M, et al. PCR on disseminated TB in bone marrow and liver biopsy specimens: Correlation to histopathological and clinical diagnosis. Scand Infect Dis 1997;29:271-4.

4 Jou NT, Yoshimori RB, Mason GR, et al. Single tube, nested, reverse transcriptase PCR for detection of viable M. tuberculosis. $\mathcal{F}$ Clin Microbiology 1997;5:1161-5.

5 Zayas R, Anguita M, Torres F, et al. Incidence of specific Zayas $R$, Anguita $M$, Torres $F$, et al. Incidence of specific aetiology and role of methods for specific aetiologic
diagnosis of primary acute pericarditis. Am 7 Cardiol 1995; 75:378-82.

6 Strang JIG, Gibson DG, Mitchison DA, et al. Controlled clinical trial of complete surgical drainage and of prednisolone in treatment of TB pericardial effusion in Transkei. Lancet 1988;ii:759-64. 